\title{
Spatial separation effects in a guiding procedure in a modified ion-beam-sputtering process
}

\author{
Sina Malobabic ${ }^{1,2}$, Marco Jupé ${ }^{1,2}$ and Detlev Ristau ${ }^{1,2}$ \\ In the present state of the art, ion beam sputtering is used to produce low-loss dielectric optics. During the manufacturing of a \\ dielectric layer stack, the deposition material must be changed, which requires rapid mechanical movement of vacuum compo- \\ nents. These mechanical components can be regarded as a risk factor for contamination during the coating process, which limits \\ the quality of high-end laser components. To minimize the particle contamination, we present a novel deposition concept that \\ does not require movable components to change the coating material during the coating process. A magnetic field guiding tech- \\ nique has been developed, which enables the tuning of the refractive index in the layer structure by sputtering mixtures with \\ varying compositions of two materials using a single-ion source. The versatility of this new concept is demonstrated for a high- \\ reflection mirror.
}

Light: Science \& Applications (2016) 5, e16044; doi:10.1038/Isa.2016.44; published online 11 March 2016

Keywords: EM field separation; ion beam sputtering; plasma guiding

\section{INTRODUCTION}

Currently, optical coating technology has reached a high level of quality with regard to optical losses and power-handling capability. However, with the development of laser systems with ever-increasing output power and beam quality, the requirements for optical coatings are continuously growing, creating a demand for new concepts for optical deposition processes. As one of the major obstacles to highquality fabrication, the particles generated in the coating process still represent a severe limiting factor for the highest-end optical components. These particles cause local absorption, increase scattering and lead to damage in short-pulse laser applications ${ }^{1}$. Therefore, the reduction of the particle densities is one of the main tasks in the current quest for optimization in thin-film production. Ion beam sputtering (IBS) is a well-established deposition process for the production of low-loss optics ${ }^{2,3}$. Although IBS is known to produce optical coatings with the lowest available levels of particle contamination, various sources of particles still exist in the process and must be considered. Various factors contribute to contamination effects. Commonly, particles are generated on the target surface during the sputtering process ${ }^{4}$. The flaking of these and other contaminating objects adhering to the fixtures in the manufacturing plant may be initiated by the mechanical movement of vacuum components such as shutters and targets, which are necessary for switching layers during the deposition process. In addition, these particles can become charged by the ion beam and be accelerated by electrostatic effects toward the substrates. A reduction of these effects can be achieved through adapted neutralization or with special arrangements of shutters and apertures. Furthermore, electromagnetic filtering concepts have been applied to reduce particle contamination. Especially for vacuum arc deposition (VAD) processes, various field guiding concepts have been developed. In VAD processes, the coating material is generated by plasma arcing at a cathode, which generates a large quantity of socalled macroparticles ${ }^{5}$. For the deposition of hard coatings on cutting tools and simple metallurgical coatings, the VAD process is sufficient. However, whereas contamination with such particles can be tolerated in these applications, the high levels of macroparticle production prevent the application of the VAD process to the more demanding areas of precision optics and electronics ${ }^{6,7}$. As early as the 1970s, the application of separation techniques such as filtered VAD (FVAD) was a well-established approach for reducing the concentration of macroparticles and, hence, enabling the adoption of the coating technique for more demanding applications in the optics and electronics industries ${ }^{5}$. The general idea of FVAD, as successfully applied by Askenov et al. ${ }^{8}$, is to use curved magnetic fields to guide the vacuum arc plasma of a metal from the source to the substrate. Unlike the conventional VAD process, the generated macroparticles cannot reach the substrate in FVAD because they are not guided along the magnetic field lines as a result of their inertia ${ }^{9}$. Consequently, the macroparticles do not become embedded in the layer structure. In most cases, the utilization of FVAD is largely confined to metallic applications such as micro-electronics. In addition, the process can be applied for the deposition of non-conductive materials, as in the case of thin films of dielectric nitrides, or semiconductors, such as amorphous silicon ${ }^{10}$, and in certain special optical applications. For example, the manufacturing of transparent conductive $\mathrm{SnO}_{2}$ films on glass has been demonstrated ${ }^{5}$. In these deposition concepts, the magnetic fields exert

${ }^{1}$ Laser Zentrum Hannover e.V., Hollerithallee 8, D-30419 Hannover, Germany and ${ }^{2}$ QUEST: Centre for Quantum Engineering and Space-Time Research, Leibniz Universität Hannover, Hannover, Germany

Correspondence: S Malobabic, Email: Sina.malobabic@web.de

Received 13 August 2015; revised 3 November 2015; accepted 10 November 2015; accepted article preview online 12 November 2015 
an influence on the trajectories of the charged material within the plasma. The fields are applied to produce collimation and guiding effects. In 1995, Anders et al. ${ }^{11}$ published their research work on FVAD, indicating that the guided plasma is displaced in the $\vec{E} \times \vec{B}$ direction, normal to the area spanned by the electric field vector $\vec{E}$ and the magnetic field vector $\vec{B}$. On the basis of this $\vec{E} \times \vec{B}$ drift, the separation of tin and titanium from a homogeneous mixture was demonstrated via delocalization of the plasma flux, leading to the separation of both species in the substrate region, by Zithomirsky et al. ${ }^{5}$. In addition, Paperny et al. ${ }^{12}$ observed the spatial separation of aluminum and titanium in a plasma-optical transport system.

On the basis of these guiding concepts, an approach for achieving low-contamination coating has been developed that uses ion beam sputtering from a target as the source of the material flux. Recent investigations have proven that the application of electromagnetic field guiding is also a very promising approach in IBS. Although common IBS processes involve a relatively low degree of ionization of the sputtered material ${ }^{13,14}$, efficient guiding by electromagnetic fields can nevertheless be achieved ${ }^{15,16}$. Related investigations have indicated that the guiding efficiency strongly depends on the plasma conditions close to the target and in the guiding tube. The guiding effect has been demonstrated for various coating materials. In a reactive process, silica, titania and alumina were deposited in an IBS-based guiding procedure. A concept for blocking particles through the application of a (bent) magnetic field separation device has recently been discussed ${ }^{17}$. In this setup, a nonlinear plasma-guiding geometry is introduced between the target and the substrate. In general, the trajectories of particles with higher masses are only marginally affected by the magnetic fields compared with those of single ions. In the cited experiments, a significant reduction in particle density was achieved because the interruption of the line of sight prevents undesired particles from reaching the substrate surface. Using this guiding system, the total number of embedded particles was reduced by a factor of three with regard to comparable layer stacks manufactured in various conventional IBS plants. In this investigation, the coating material was changed by translating the zone target ${ }^{17}$. According to the approach of Zhitomirsky, a coating process without any movement of mechanical components should be possible because the mechanical movement during the changing of the coating material can be replaced with a purposeful variation in the magnetic fields to select the desired coating material.

The investigations presented here were focused on the manufacturing of complex dielectric layer stacks. Consequently, the first part of the study consisted of an investigation of the influence of the magnetic field strength on the lateral thickness distribution for the binary oxide titania, before the background of the dynamics in the plasma flux during the guiding process was considered. As a second step, the local distribution during the deposition of ternary layers via magnetic field guiding was investigated. Furthermore, the influence of the magnetic field strength and the field polarization were analyzed. The process parameters were optimized for a predefined ternary mixture of alumina and titania with respect to the maximum achievable contrast in the refractive index, with the application of magnetic field separation.

Finally, the production of a high-reflection stack was demonstrated using the presented concept of a material flux that is fully controlled by magnetic fields.

\section{MATERIALS AND METHODS}

\section{Setup and trajectory manipulation}

The setup, including the magnetic field separator used for the investigations, is depicted in Figure 1. In general, the coating process was based on a common IBS co-sputtering process. In accordance with the typical IBS principle, the process chamber was equipped with a high-power ion source (Veeco $16 \mathrm{~cm}$ High Power) combined with a neutralizer (electron emitter). The coating material was sputtered from a zone target, which consisted of two pure metals, aluminum and titanium. The concentrations of each in the mixed material flux were defined by the area coverage of the ion beam on the aluminum and titanium zones, and the composition of the mixture could be varied by means of linear movements of the zone target perpendicular to the ion beam. In general, the parameters of the IBS process were held constant using 20 s.c.c.m. of activated oxygen in a reactive process. During the experiments, the $\mathrm{O}_{2}$ was injected through the ion source and, consequently, was partially ionized. The source was operated at an ion current of $300 \mathrm{~mA}$ and a beam voltage of $800 \mathrm{~V}$ during the experiments. In addition to these common features of the cosputtering process, a magnetic field separator was also included in the setup.

The separator consisted of a double-wound, closely spaced coil that could produce an axial magnetic field strength of up to $60 \mathrm{mT}$ inside the coil at a maximum coil current of 340 A. The coil was wound on a cylindrical glass tube, which provided sufficient stability. The axis of symmetry of the coil was tilted by $20^{\circ}$ with respect to the target normal, and the distance between the target and coil was $10 \mathrm{~cm}$. In total, the path length from the target surface to the substrate area at the exit of the coil was equal to $40 \mathrm{~cm}$, including the $30 \mathrm{~cm}$ length of the guiding coil (Figure 1). The coil was positioned at the maximum of the sputter distribution such that the majority of the material was directed toward the coil aperture. The magnetic field was varied proportionally to the coil current in a range of -340 to $+340 \mathrm{~A}$.

For a more comprehensive discussion of the flux of deposition material in the separator, the generated magnetic field profile must be considered.
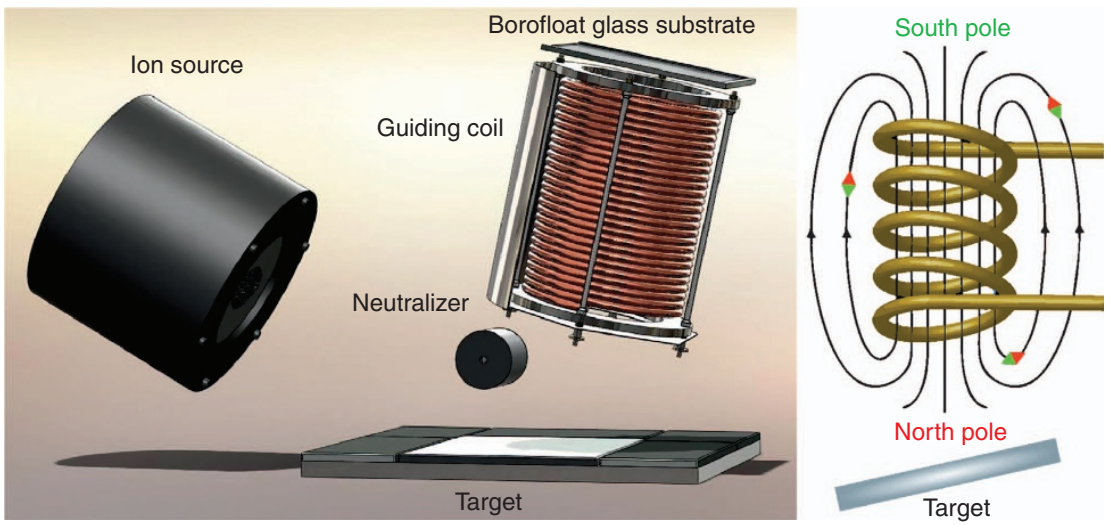

Figure 1 Setup of the separator and schematic distribution of the magnetic field lines $\left(\vec{B} \downarrow\right.$ and $\left.\vec{v}_{\text {elec }} \uparrow\right)$. 
The guiding device provides a low magnetic field strength at the outer region of the coil. The magnetic field intensifies at the entrance of the device, and the field lines taper toward it. Inside the coil, the magnetic field is homogenous and axially aligned; thus, the magnetic field forms equipotential lines that provide high longitudinal guiding of the plasma. This magnetic field can be written in cylindrical coordinates as $\vec{B}=\left(0,0, B_{z}\right)$.

The sputtering process must also be incorporated into this picture. An ion source provides an argon plasma beam to sputter the deposition material from the target. In a typical IBS process, the sputtered species exist predominantly in a state of electrical neutrality. The typical ratios of charged to uncharged sputtered material are estimated to lie in a range from $10^{-413}$ up to a small percentage, depending on the composition and configuration of the target (metal or oxide) and the neutralization system ${ }^{14}$. This small degree of ionization also depends on the plasma conditions and the material.

For efficient guiding, the coating material must be ionized to some extent. Because the ionization rate produced in the sputtering process is relatively low, other ionization mechanisms, such as charge transfer or impact ionization, must be considered for the guiding of ion-beam-sputtered species. In the present study, a major contribution from ionization induced by collision with electrons in the plasma region at the entrance of the guiding coil was expected. To specify the total kinetic behavior of the plasma, including ionization, charge transfer, absorption and desorption must all be assessed. In a qualitative approach to the description of the ionization effect, a discussion of the electron trajectories is of the highest priority. The electrons move along the magnetic field lines as a result of the Lorentz force. Because the electrons have a velocity component parallel to the axial magnetic field, they are forced into circular trajectories and are guided through the solenoid. The radius of their circular trajectories can be approximated by the Larmor radius ${ }^{18}$ :

$$
r_{\text {Larmor }}=m v_{\perp} /(q|\vec{B}|)
$$

where $m$ is the mass, $v_{\perp}$ is the velocity, $|\vec{B}|$ is the amplitude of the magnetic field vector and $q$ is the charge of the particle. This confinement is often referred to as the phenomenon of magnetized electrons ${ }^{19}$. As they travel toward the entrance to the coil, the electrons pass through an inhomogeneous magnetic field because they are streaming into a region of higher magnetic field strength ${ }^{11}$. A small fraction of the electrons are partially reflected as a result of the magnetic mirror effect ${ }^{20,21}$. The majority of the gyrating electrons enter the internal region of the solenoid, where the magnetic field lines are parallel to the solenoid (Figure 1), and their Larmor radius is smaller than the radius of the solenoid. By contrast, the trajectories of the material ions are barely influenced by the magnetic field as a consequence of their much higher masses.

This electron confinement leads to a separation of ions and electrons in the plasma and creates a radial electric field that points toward the central axis of the coil. In this model, the movement of the ions is induced by their electrical attraction to the electrons that are gyrating around and radially trapped along the magnetic field lines as they are guided through the solenoid ${ }^{22,23}$. As confirmed by Zhitomirsky et al. the resulting electric field is responsible for the guiding effect of the plasma. In this scenario, the electrostatic forces keep the electrons and ions together. This applies also to the rotation of the plasma, which is governed by the well-known $\vec{E} \times \vec{B}$ drift motion. The ions and electrons drift in the same direction, perpendicular to the electric field $\vec{E}$ and the magnetic field $\vec{B}$, with a velocity

$$
v_{\perp}=\frac{(\vec{E} \times \vec{B})}{|\vec{B}|^{2}}
$$

inducing a rotation of the plasma ${ }^{11}$. The rotation frequency $\omega$ is proportional to the magnetic field, and the radius $r_{0}$ of the plasma column is inversely proportional to the magnetic field, $r_{0} \sim 1 / B^{24}$. Geva et al. introduced a steadystate fluid model of a rotating multispecies plasma ${ }^{25}$. They noted that in the steady state, although the different ions rotate at the same frequency, the electron frequency is slightly different from the ion frequency. The azimuthal diamagnetic current due to the differential motion of the electron and ions is responsible for the radial confining force acting on the ions against the centrifugal force and pressure gradient, which act radially outwards. Neglecting the azimuthal and axial gradients, the radial components of the momentum equation for the electrons in a cylindrical coordinate system with $\vec{B}=$ $\left(0,0, B_{z}\right)$ can be written as

$$
\underbrace{\frac{-M_{e} V_{\theta e}^{2}}{r}}_{\text {Centrifugal force }}=\underbrace{-e\left(E_{r}+V_{\theta e} B_{z}\right)}_{\text {Lorentz force }}-\underbrace{k T \frac{\mathrm{d}}{\mathrm{d} r}\left(\ln \frac{n_{e}}{n_{0}}\right)}_{\text {Pressure gradient }}
$$

For the ions, a similar relation applies:

$$
\underbrace{\frac{-M_{i} V_{\theta i}^{2}}{r}}_{\text {Centrifugal force }}=\underbrace{Z_{i}\left(E_{r}+V_{\theta i} B_{z}\right)}_{\text {Lorentz force }}-\underbrace{k T \frac{\mathrm{d}}{\mathrm{d} r}\left(\ln \frac{n_{i}}{n_{0}}\right)}_{\text {Pressure gradient }}
$$

where $M_{e}$ and $M_{i}$ are the masses, $e$ is the elementary charge on an electron and $Z_{i}$ is the ion charge of species $i$. Moreover, $n_{\mathrm{i}}$ and $n_{\mathrm{e}}$ denote the densities of ions and electrons, respectively, and $n_{0}$ is the particle density of the total ensemble. In a rigid-rotor equilibrium state $V_{\theta i}=\omega_{i} r$, where $V_{\theta i}$ is the tangential velocity, the pressure $p_{i}$ of each ion species $i$ is assumed to be scalar and expressed via an equation of state as $p_{i}=n_{i} k T$, where $k$ is the Boltzmann constant and $T$ is the temperature, which can be approximated as $1 \mathrm{eV}$ according to Geva et al. Upon solving for $n_{i}$ and integrating between $r=0$ and $r=R$, where $R$ is the radius of the coil, Equation (4) yields

$$
n_{i}(r)=n_{i}(0) \exp \left[\frac{Z_{i} e r^{2}}{2 k T}\left(\omega_{i} B_{z}+\frac{M_{i} \omega_{i}^{2}}{Z_{i} e}\right)+\frac{Z_{i} e}{k T} \int_{0}^{R} E_{r}(r) \mathrm{d} r\right]
$$

From this equation, one can derive the separation factor ${ }^{25}$ that arises from the stationary rotation of the plasma and leads to a centrifugal, radial separation between particles of different mass/charge ratios:

$$
S=\frac{\left[n_{i}(r)\right]^{1 / Z_{i}} /\left[n_{j}(r)\right]^{1 / Z_{j}}}{\left[n_{i}(0)\right]^{1 / Z_{i}} /\left[n_{j}(0)\right]^{1 / Z_{j}}}=\exp \left[\frac{\omega^{2} r^{2}}{2 k T}\left(\frac{M_{i}}{Z_{i}}-\frac{M_{j}}{Z_{j}}\right)\right]
$$

At a given radial distance in the plasma column, the separation depends exponentially on $\omega^{2}$, and thus, because $\omega \propto B$, the separation scales exponentially with $B^{224}$. Consequently, an effective separation of titanium and aluminum atoms should be achieved during the guiding process. Notably, because of the plasma-guiding effect, the rotation frequency is assumed to be constant for different species.

In summary, the trapping of the electrons along the center line of the solenoid should not only cause a guiding of the ions but also lead to a rotation of the plasma and the separation of its constituent species as it is guided through the solenoid ${ }^{2-28}$. The model of Geva et al. offers a simple picture of the separation of a homogenous mixture. Because of the more complex dynamics of the IBS-driven guiding process, however, this model cannot describe the separation in full detail, and some differences are expected.

\section{RESULTS AND DISCUSSION}

\section{Spatial separation/guiding of $\mathrm{TiO}_{2}$}

The analysis of the material flux dynamics during the guiding process is an important issue. To this end, preliminary investigations of the magnetic-field-induced variations in the lateral coating material distributions were performed. As a first step, the lateral distributions of titania thin films deposited on Borofloat substrates $\left(230 \times 230 \times 1 \mathrm{~mm}^{3}\right)$ at the exit of the linear magnetic guiding tube were determined based on spectrophotometric measurements. In this measurement series, the magnetic field strength and the field polarization were varied by applying stepwise changes to the coil current $\left(I_{\text {Coil }}=0, \pm 50, \pm 100, \pm 200, \pm 300\right.$ and $\left.\pm 340 \mathrm{~A}\right)$. Here a positive polarization is assigned to states in which the plasma flux is parallel to the magnetic field direction $\left(\vec{B} \uparrow\right.$ and $\left.\vec{v}_{\text {elec }} \uparrow\right)$, and a negative polarization corresponds to a plasma flux that is antiparallel to the magnetic field direction $\left(\vec{B} \downarrow\right.$ and $\left.\vec{v}_{\text {elec }} \uparrow\right)$.

Figure 2 displays the results of coating runs with different field strengths and polarizations. Photographs of the samples are presented in Figure 2a. The upper row summarizes the lateral distributions of the samples that were coated with a positive polarization, whereas the 
a

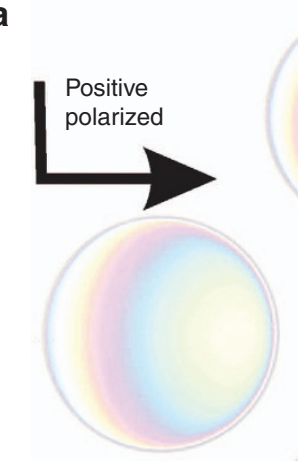

Negative

polarized

polarized

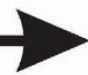

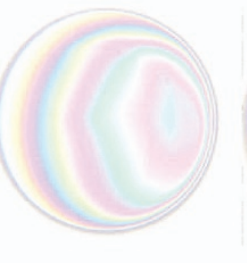
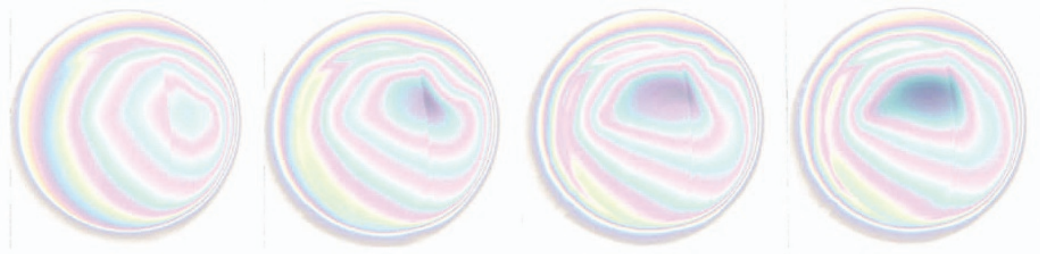

$50 \mathrm{~A}$

$100 \mathrm{~A}$

$200 \mathrm{~A}$

$300 \mathrm{~A}$

$340 \mathrm{~A}$
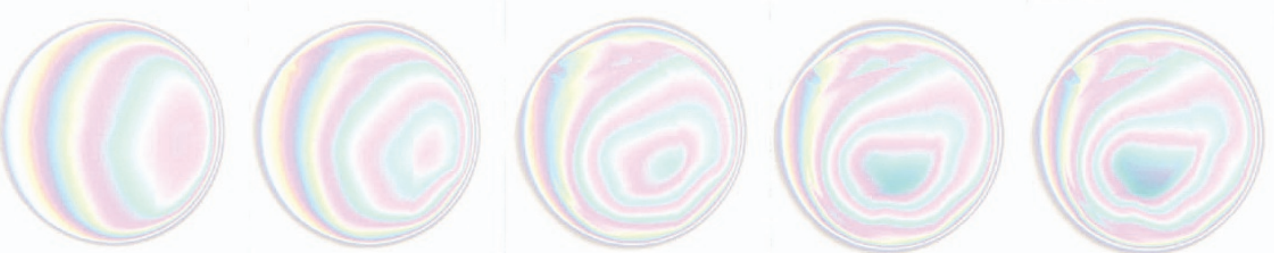

b

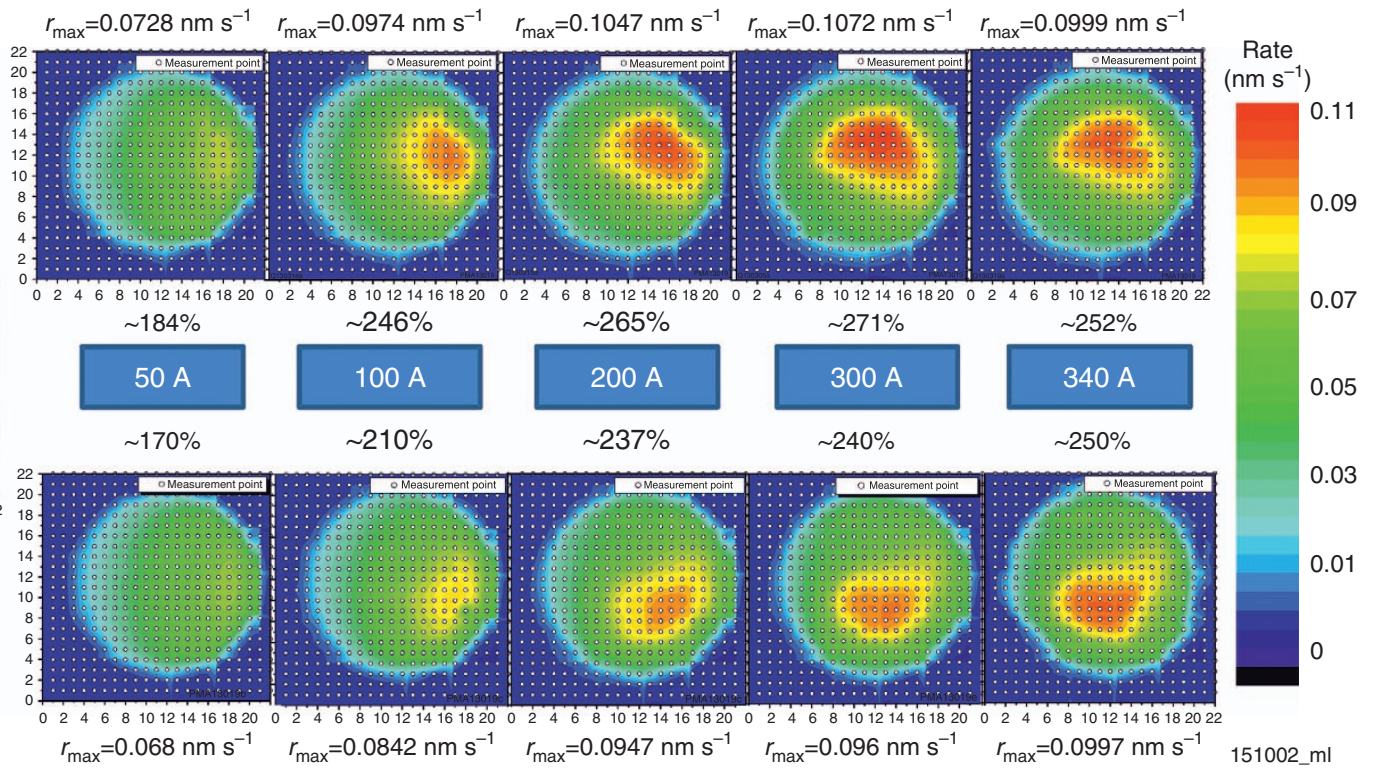

Figure 2 Lateral distributions of $\mathrm{TiO}_{2}$ on Borofloat substrates by applying different coil currents. (a) Photocopies of the coated Borofloat substrates. (b) Lateral distribution of deposition rates determined by evaluation of spectrophotometric measurements.

samples in the bottom row were coated with a negative polarization of the magnetic field. In the absence of a magnetic field, the lateral distribution is influenced by a shadowing effect of the coating material caused by the shape of the solenoid and the angle with respect to the target normal. The material distributions in the photographs clearly indicate a rotation around a longitudinal axis, which is induced by the electron confinement. The angle of rotation depends on the magnetic field strength, and the rotation direction is inverted upon the inversion of the magnetic field polarization. The rotation of the plasma is clockwise in the case of negative polarization and counterclockwise in the case of positive polarization. This change in the lateral distribution and the observed behavior of the rotation angles are in accordance with previous results measured for alumina coatings deposited under the influence of magnetic field guiding in a similar IBS process ${ }^{16}$.

According to Boxman et al. ${ }^{19}$, the rotation of the plasma flux can be traced back to the $\vec{E} \times \vec{B}$ drift. The present observations are also in agreement with previous studies by Andre et al., who investigated a
VAD process in connection with a bent magnetic field separator for the application of metallic aluminum ${ }^{11}$.

The lateral distributions were quantitatively determined from matrices of spectrophotometric measurements acquired at $22 \times 22$ test positions on the samples. The evaluations of the lateral thickness distributions are displayed in Figure 2b. Regarding the polarization and the magnetic field strength, the thickness plots are arranged in the same manner as the photographs in Figure 2a. Generally, the lateral deposition rate has a well-defined maximum at a characteristic position. The rate decreases with increasing distance from this maximum, and the behavior can be approximated by two Gaussian distributions, one each in the $x$ and $y$ directions ${ }^{11}$. Obviously, both the position of the maximum and the deposition rate depend on the magnetic field and the polarization. As shown in the photographs, the maximum rotates around the center line of the coil. In Figure 2b, the deposition rate is normalized in units of percent with respect to the reference layer deposited without any magnetic field. The local 
maximum deposition rate in the absence of a magnetic field was determined to be $0.039 \mathrm{~nm} \mathrm{~s}^{-1}$. Higher magnetic fields lead to a local increase in the guiding efficiency for the coating material. A maximum deposition rate of $0.1072 \mathrm{~nm} \mathrm{~s}^{-1}$ was observed for a coil current of $300 \mathrm{~A}$ and a positive polarization.

Consequently, an increase in the deposition rate of approximately a factor of three could be achieved. In this context, the polarization also influences the guiding efficiency. In this study, the gain in material transport was lower for an antiparallel orientation of the magnetic field with respect to the material flux. In summary, the application of a stronger magnetic field improved the guiding effect and increased the local deposition rate, as shown in the upper row of Figure $2 b$.

\section{Spatial separation of a $\mathrm{TiO}_{2} / \mathrm{Al}_{2} \mathrm{O}_{3}$ composite}

This section discusses an investigation of the influence of the guiding technique on different material species. The observed rotations depend on the atomic masses of the different materials ${ }^{5}$. This effect should cause a lateral separation of species with different mass-to-charge ratios on the substrate plane ${ }^{29}$. To study this separation effect, a mixed material flow was created through the IBS co-sputtering of titanium and aluminum from a zone target ${ }^{30}$. The material composition was defined by the ion beam coverage, which was adjusted such that $70 \%$ of its area was incident on the titanium zone and 30\% was incident on aluminum. The magnetic field strength of the linear separator and the field polarization were varied by applying stepwise changes to the coil current, $I_{\text {Coil }}=0, \pm 50, \pm 100, \pm 200$ and \pm 340 A. For the analysis of the material flux, single layers on Borofloat substrates were evaluated with respect to their lateral compositions, their refractive indices and their film thickness distributions.

The results of the various coating series are summarized in Figure 3. First, a correlation between an energy-dispersive X-ray analysis of the ternary mixtures and the compositional data extracted from the refractive indices was established. Good agreement was observed for the material compositions of alumina and titania. Therefore, in subsequent experiments, the compositions of the mixtures were determined based on the indices of refraction. Obviously, as indicated by the specific refractive indices of the deposited coatings displayed in Figure 3a, the magnetic field strength influences the distribution of the material on the substrate plane and leads to local concentrations of particular species. Thus, the electromagnetic field separates the species in the coating material flux as it passes through the linear coil and accordingly causes different concentrations of components to form at the exit. According to Gidalevich et al. ${ }^{31}$, the resulting refractive index patterns can be traced back to the increasing azimuthal plasma drift in the crossed axial magnetic and radial electric fields, which affects the different species in different ways. Geva et al. explained the phenomenon in terms of a centrifugal force that creates a radial separation between particles of different mass/charge ratios ${ }^{25}$.

A more detailed interpretation of the $\mathrm{Al}_{2} \mathrm{O}_{3}$ concentrations presented in Figure 3a reveals that the magnetic field leads to a local separation of the composite coating material. In addition, the global concentration of the mixture over the entire sample is also influenced by the magnetic field. The average global concentration can be calculated by considering the film thicknesses and the local concentrations. In this manner, it can be found that for a negatively polarized magnetic field, the weighted average composition consisted of $68.3 \%$ aluminum; this is significantly higher than the corresponding value of $53.4 \%$ obtained using a positively polarized magnetic field.

This phenomenon can be attributed to the current of electrons attracted by the coil. The electrons trapped in the coil are assumed to be responsible for the ionization of the neutral sputtered coating
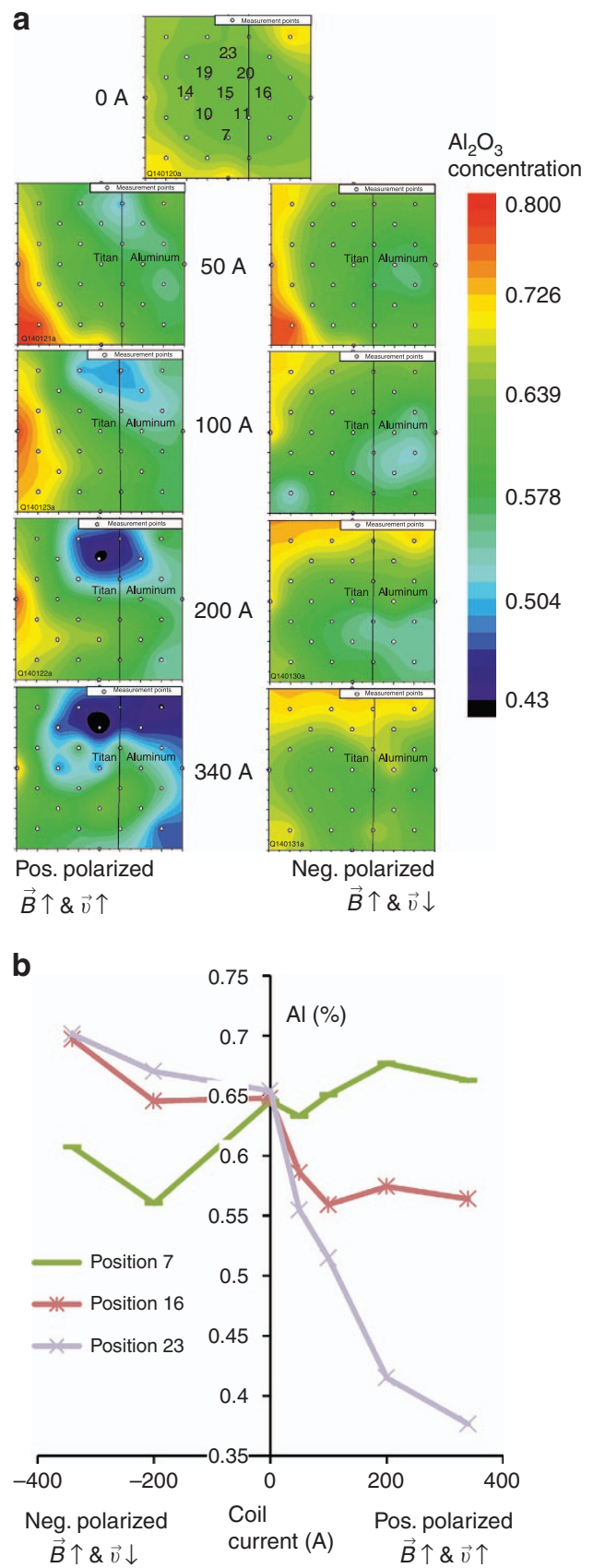

Figure 3 (a) Lateral refractive index distributions of coatings produced by sputtering a composite of $\mathrm{Al}_{x} \mathrm{Ti}_{y} \mathrm{O}$ onto Borofloat substrates (the vertical black line represents the projection of the conjugation line of both target materials). (b) Relevant positions at which a sufficient deposition rate could be achieved.

material. Generally, the movement of the highly energetic electrons inside the vacuum chamber is defined by the magnetic field lines. With a change in the polarization of the coil current, the electron trajectories are spatially flipped because of the $\overrightarrow{\boldsymbol{E}} \times \overrightarrow{\boldsymbol{B}}$ drift described above and the plasma rotation is reversed ${ }^{25}$. This effect influences both the guiding and the ionization of the coating material. When ionization occurs above the titanium target, the shift causes a displacement of the spatial ionization toward the aluminum target.

Generally, an increase in the magnetic field strength intensifies the concentration of the corresponding species, enabling the tuning of the 
refractive indices at specific locations. In a simple approach, a relatively strong local index can be achieved by reversing the magnetic field at the maximum coil current. This effect is illustrated in Figure $3 \mathrm{~b}$, which depicts the variations in the refractive indices at selected locations on the substrate plane as a function of the magnetic coil current. Before the testing of the intended production technique for application in practicable coating systems, these positions were selected as suitable based on the ability to achieve high deposition rates.

To achieve the highest possible variation in concentration by means of the separation technique and, consequently, the highest contrast in the index of refraction, a further study addressing different ion beam area coverages of the zone target was performed. In Figure 4, the material composition at position 23 (Figure 3a), with a high deposition rate on the substrate plane, as a function of the coil current is depicted for selected Ti/Al coverage ratios of 70/30, 50/50 and $30 / 70$. For simplicity, only the aluminum concentration is considered for the three coil current values of 0 and $\pm 300 \mathrm{~A}$. The variations in the initial mixtures of the sputtered material in the absence of a magnetic field indicate a higher sputtering efficiency for aluminum than for titanium. Consequently, the composition of the sputtered material flux was not directly equivalent to the ratio of the material areas covered by the ion beam on the target. For example, a ratio of $30 / 70$ resulted in a mixture of nearly $88 \%$ alumina, a 50/50 ratio yielded $80 \%$ alumina and a coverage ratio of $70 / 30$ still produced $65 \%$ alumina at the substrate. It is assumed that a sputter zone ratio of $\sim 90 / 10$ would yield a 50/50 composition on the substrate plane. This coverage ratio would lead to a significant increase in the absorption of the layer (Figure 2) and was not suitable for further investigations. However, a nearly balanced composition of the two coating materials could be observed upon sputtering the species on the target in a ratio of $70 \%$ titanium and $30 \%$ aluminum. In this case, the concentration of each species at the substrate yielded the optimal material

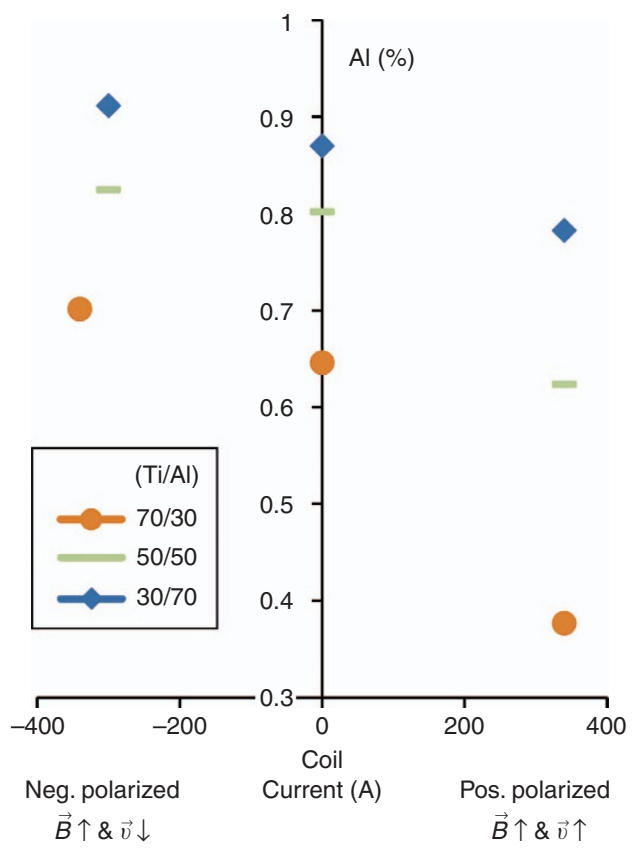

Figure 4 Concentrations of aluminum at position 23 as a function of the ion beam coverage on the target and the coil current. distribution and resulted in the highest refractive index contrast under the application of a $\pm 300 \mathrm{~A}$ coil current, as seen in Figure 4.

However, with the application of magnetic field guiding, this ratio is influenced by the ionization energies of the different species; titanium has an ionization energy of $658.1 \mathrm{~kJ} \mathrm{~mol}^{-1}$ and that of aluminum is $577.5 \mathrm{~kJ} \mathrm{~mol}^{-1}$. Therefore, for equal contents of both materials, a higher quantity of alumina is ionized and interacts with the magnetic field. Furthermore, the material concentration can also be influenced by the guiding efficiency.

In summary, the presented concept enables not only the spatial manipulation of the local thickness of optical layers (Figure 2) but also the adjustment of the local $\mathrm{TiO}_{2} / \mathrm{Al}_{2} \mathrm{O}_{3}$ ratio and, consequently, the refractive indices (Figure 3 ). This opens a new route toward the production of optical layers with different ratios of $\mathrm{TiO}_{2} / \mathrm{Al}_{2} \mathrm{O}_{3}$ and different local refractive indices without the need to switch sputter targets. Consequently, magnetic fields may be applied to separate the species in the coating material and accordingly create different concentrations of components in optical layers.

\section{Production of a high-reflection mirror}

Usually, in addition to a high deposition rate, the highest possible refractive index contrast is advantageous for the production of highreflection mirrors. It has been shown that the position on the substrate at which the maximum deposition rate is achieved can be controlled by adjusting the polarization of the magnetic field. In addition to the rate, the concentration at a defined position can also be controlled by tuning the magnetic field of the separator. In this context, position 23 (Figure 3a) was observed to offer a high deposition rate in conjunction with a sufficiently high refractive index contrast (Figure 3b). Figure 5 displays the dispersion curves obtained at position 23 using a magnetic field generated at coil currents of 250 and $-250 \mathrm{~A}$ for different ion beam Ti/Al area coverage ratios of 70/30, 50/50 and 30/70 on the zone target. Because the 70/30 ratio resulted in the highest refractive index contrast, this arrangement was used for the subsequent single-layer investigations and to demonstrate the production of a high-reflection mirror. Because the lateral distribution of the deposition rate exhibits an approximately Gaussian profile, a rotating substrate holder was installed to achieve sufficient lateral homogeneity of the highreflection mirror.

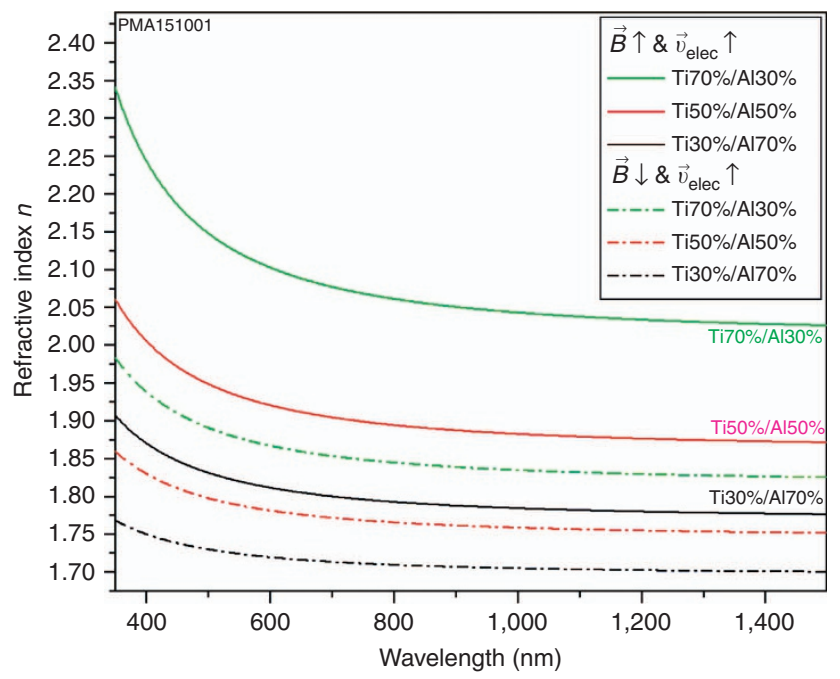

Figure 5 Dispersion curves of TiAlO layers produced using initial Ti/Al coverage ratios of $70 / 30,50 / 50$ and $30 / 70$ on the zone target with negative and positive polarizations of the magnetic field. 


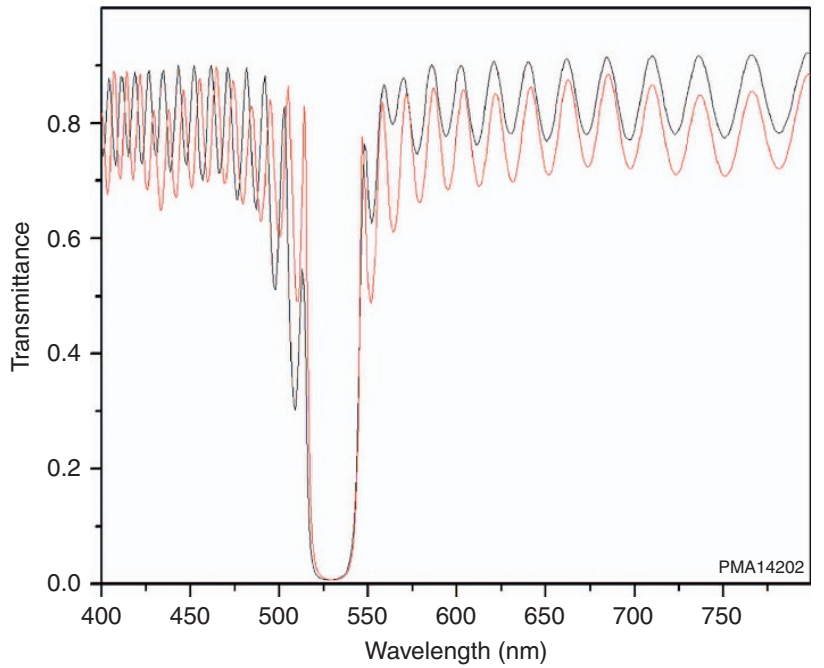

Figure 6 Optical spectrum of the coated high-reflection mirror fabricated using the separator device.

The deposition rates at the sample must be determined through ex situ measurements of single-layer coatings. Therefore, in a preliminary study, single layers of various mixtures were coated on Suprasil substrates, and the refractive indices and deposition rates of both the low-refractive-index and high-refractive-index layers were determined through the evaluation of spectrophotometric measurements. A positive polarization $(+250 \mathrm{~A})$ yielded a high refractive index of 2.00, and a negative polarization $(-250 \mathrm{~A})$ yielded a low refractive index of 1.88. A coating rate of $0.114 \mathrm{~nm} \mathrm{~s}^{-1}$ was achieved by applying a positively polarized magnetic field, whereas a coating rate of $0.120 \mathrm{~nm} \mathrm{~s}^{-1}$ was achieved by applying a negatively polarized magnetic field. In total, the measured rates indicated that the times required for the deposition of quarter-wave optical thickness layers were $558 \mathrm{~s}$ for a low-index layer and $594 \mathrm{~s}$ for a high-index layer. Under these conditions, beginning with the high-index material, a Suprasil substrate was coated with 71 alternating layers by manually reversing the magnetic field over a duration of $\sim 13 \mathrm{~h}$. The spectrum of the coated mirror is shown in Figure 6; this spectrum deviates from the ideal spectrum in the range of $>560 \mathrm{~nm}$ and does not exhibit a central wavelength of exactly $532 \mathrm{~nm}$, but in general, the highreflection mirror fulfills the performance requirements.

\section{CONCLUSION}

The effects of magnetic fields on the distribution of coating materials in IBS were investigated. During the sputtering of a binary material, magnetic fields can be applied to generate a guiding effect that causes a rotation of the position of the local rate maximum in addition to a gain in the overall deposition rate. When using a mixed flux in which the plasma contains at least two species, the magnetic fields additionally cause a separation of the species, leading to different local material compositions on the substrate plane. Consequently, the material composition and, accordingly, the refractive index of the coating at certain positions can be adjusted by tuning the magnetic field of the separator. On the basis of this effect, layered systems with refractive indices that vary with depth can be produced without any mechanical movements in the deposition system by simply tuning the magnetic field by adjusting the coil current. This novel deposition concept was successfully applied in the production of a high-reflection mirror for a wavelength of $532 \mathrm{~nm}$ by alternating the polarization of the magnetic field.
In conclusion, the production of multilayer optical coating systems without the need for moving parts in the deposition system was demonstrated for the first time on the basis of phase separation. The developed ion-beam-sputtering process, which uses a zone target and a magnetic separator, opens a novel route for the deposition of highquality optical coating systems with reduced particle contamination. Especially in high-power applications, undesired particles may limit the laser-induced damage thresholds of coated optics elements because they act as initial seeds in the thermal damage process. In addition, the performance of filters with high optical density is often degraded as a consequence of particle-related pinhole formation in their coatings. In many cases, embedded particles also have detrimental effects on the environmental stability of optical coatings and their resistance to mechanical or chemical effects. Finally, the reduction of the defect density in coatings can improve their optical loss properties, which are of the utmost importance in high-finesse applications. Consequently, the implementation of filtered ion beam sputtering is a promising technology for the improvement of optical coatings and various highend components.

\section{CONFLICT OF INTEREST}

The authors declare no conflict of interest.

\section{ACKNOWLEDGEMENTS}

This work was supported by the Deutsche Forschungsgemeinschaft (DFG) within the framework of the Cluster of Excellence QUEST Project 201.

1 Ristau D. Laser-induced Damage in Optical Materials. Boca Raton, FL: CRC Press; 2014.

2 Cimma B, Forest D, Ganau P, Lagrange B, Mackowski JM et al. Ion beam sputtering coatings on large substrates: toward an improvement of the mechanical and optical performances. Appl Opt 2006; 45: 1436-1439.

3 Flaminio R, Franc J, Michel C, Morgado N, Pinard L et al. A study of coating mechanical and optical losses in view of reducing mirror thermal noise in gravitational wave detectors. Class Quant Grav 2010; 27: 084030.

4 Walton CC, Kearney PA, Folta JA, Sweeney DW, Mirkarimi PB. Understanding particle defect transport in an ultra clean sputter coating process. In: Engelstad RL (ed), Proceedings of Emerging Lithographic Technologies VIII, 23 February 2003; Santa Clara, CA, USA. SPIE Publishing: Santa Clara, CA, USA, 2003, p 470.

5 Zhitomirsky VN, Kaplan L, Boxman RL, Goldsmith S. Ion current distribution in filtered vacuum arc deposition system. Surf Coat Technol 1995; 76-77: 190-196.

6 Martin PJ, Bendavid A. Review of the filtered vacuum arc process and materials deposition. Thin Solid Films 2001; 394: 1-14.

7 Boxman RL, Sanders DM, Martin PJ. Handbook of Vacuum Arc Science and Technology: Fundamentals and Applications. William Andrew; 1995.

8 Aksenov II, Belous VA, Padalka VG, Khoroshikh VM. Transport of plasma streams in a curvilinear plasma-optics system. Sov J Plasma Phys 1978; 4: 758-763.

9 Shi X, Tay BK, Tan HS, Liu E, Shi J et al. Transport of vacuum arc plasma through an off-plane double bend filtering duct. Thin Solid Films 1999; 345: 1-6.

10 Arbilly D, Boxman RL, Goldsmith S, Rothwarf A, Kaplan L. Amorphous Si thin films prepared by vacuum arc deposition. Thin Solid Films 1994; 253: 62-66.

11 Anders A, Anders S, Brown IG. Transport of vacuum arc plasmas through magnetic macroparticle filters. Plasma Sour Sci Technol 1995; 4: 1.

12 Paperny VL, Krasov VI, Chrnich AA, Lebedev NV, Astrakchantsev NV. Transport of plasma fluxes with distinct ion masses through a curved magnetic field. In: Proceedings of the 24th International Symposium on Discharges and Electrical Insulation in Vacuum. IEEE: Braunschweig, Germany; 2010, pp 332-334.

13 Betz G, Wien K. Energy and angular distributions of sputtered particles. Int J Mass Spectrom Ion Processes 1994; 140: 1-110.

14 van der Weg WF, Rol PK. On the excited state of sputtered particles. Nucl Instrum Methods 1965; 38: 274-276.

15 Malobabic S, Jupé M, Ristau D. Towards an electro-magnetic field separation of deposited material implemented in an ion beam sputter process. Appl Phys Lett 2013; 22: 221604

16 Malobabic S, Jupé M, Ristau D. Investigations in the guiding efficiency in a modified ion beam sputtering process. App/ Opt 2013; 52: 8212-8219.

17 Malobabic S, Jupé M, Kadhkoda P, Ristau D. Towards a magnetic field separation in ion beam sputtering processes. Thin Solid Films 2015; 592: 271-275.

18 Lieberman MA, Lichtenberg AJ. Principles of Plasma Discharges and Materials Processing. New York: Wiley; 1994. 
19 Boxman RL, Zhitomirsky V, Alterkop B, Gidalevich E, Beilis I et al. Recent progress in filtered vacuum arc deposition. Surf Coat Technol 1996; 86-87: 243-253.

20 Anders S, Anders A, Brown I. Focused injection of vacuum arc plasmas into curved magnetic filters. J Appl Phys 1994; 75: 4895-4899.

21 Chen FF. Introduction to Plasma Physics. New York: Plenum; 1974.

22 Alterkop B, Gidalevich E, Goldsmith S, Boxman RL. Vacuum arc plasma jet propagation in a toroidal duct. J Appl Phys 1996; 79: 6791.

23 Sanders DM, Anders A. Review of cathodic arc deposition technology at the start of the new millennium. Surf Coat Technol 2000; 133-134: 78-90.

24 Prasad RR, Krishnan M. Scaling of rotation and isotope separation in a vacuum-arc centrifuge. Nucl Instrum Methods Phys Res Sect B 1987; 26: 65-71.

25 Geva M, Krishnan M, Hirshfield JL. Element and isotope separation in a vacuum-arc centrifuge. J Appl Phys 1984; 56: 1398-1413.

26 Geva M, Cohen C, Danziger O, Dothan F, Friedland L et al. Vacuum arc plasma centrifuge for element and isotope separation. IEEE Trans Plasma Sci 1987; 15: 583-588.

27 Kaneko O, Sasaki S, Kawashima N. Mass separation experiment with a partially ionized rotating plasma. Plasma Phys 1978; 20: 1167.

28 Giuliani L, Grondona D, Kelly H, Minotti FO. On the plasma rotation in a straight magnetized filter of a pulsed vacuum arc. J Phys D: Appl Phys 2007; 40: 401.
29 Geva M, Krishnan M, Hirshfield JL. Centrifugal mass separation in laser-initiated vacuum arcs. Nucl Instrum Methods Phys Res 1981; 186: 183-187.

30 Lappschies M, Görtz B, Ristau D. Application of optical broad band monitoring to quasi-rugate filters by ion beam sputtering. Appl Opt 2006; 45: 1502-1506.

31 Gidalevich E, Goldsmith S, Boxman RL. The role of the electrical polarization field in magnetic plasma confinement. J Phys D: Appl Phys 2003; 36: 653.

(c) (1) () $\odot$ This work is licensed under a Creative Commons Attribution-NonCommercial-NoDerivs 4.0 International License. The images or other third party material in this article are included in the article's Creative Commons license, unless indicated otherwise in the credit line; if the material is not included under the Creative Commons license, users will need to obtain permission from the license holder to reproduce the material. To view a copy of this license, visit http://creativecommons.org/licenses/by-nc-nd/4.0/ 